endocrine and immune dysfunction, mood disorders, tolerance, addiction and death. Our systematic review estimated an incidence of iatrogenic misuse or addiction of $\sim 4 \%$ among all people prescribed an opioid. The other problem is with the lack of high quality evidence to support their effectiveness in the long-term relief of pain, as we will hear later. These concerns have prompted the International Association for the Study of Pain to issue a recent carefully-worded statement on the use of opioids in chronic pain, advising caution and close monitoring. This presentation will summarise these issues, and present evidence of the increasing use of opioids in recent years, focusing on Europe and particularly Scotland, where prescribing rates rose $65 \%$ in a 10 year period. I will present evidence of both good and potentially harmful prescribing, evidence that most prescribing is "appropriate" in that it is associated with severe chronic pain, and, despite this, that a very large proportion of people with chronic pain are not prescribed any opioid. Opioids may still have a role in chronic non-malignant pain, but the goals of their use need to be increased function and improved quality of life, and we need careful selection of patients and evaluation of outcomes.

Disclosure of Interest: B. Smith Grant/research support from: Scottish Government, Chief Scientist Office

DOI: 10.1136/annrheumdis-2018-eular.7668

\section{SP0074 ARE ALL OPIOIDS THE SAME?}

C. Stein. Anesthesiology and Intensive Care Medicine, Charité - Freie Universität Berlin, Berlin, Germany

Indiscriminate activation of opioid receptors provides pain relief but also severe central and intestinal side effects such as respiratory depression, sedation, addiction, nausea and constipation. Novel strategies to avoid adverse effects include the design of "biassed agonists" and of environment-sensitive ligands. Exploiting pathological (rather than physiological) conformation dynamics of opioid receptorligand interactions might yield ligands without adverse actions. By computer simulations at low $\mathrm{pH}$, a hallmark of injured tissue, we designed an agonist that, due to its low pKa, selectively activates peripheral mu-opioid receptors in injured/ inflamed tissue, i.e. at the source of pain generation. Unlike conventional opioids, this agonist showed $\mathrm{pH}$-sensitive binding, G-protein subunit dissociation, and cAMP inhibition in vitro. It produced injury-restricted analgesia in rats with different types of inflammatory pain without exhibiting respiratory depression, sedation, constipation or addiction potential.

Disclosure of Interest: None declared

DOI: 10.1136/annrheumdis-2018-eular.7740

\section{THURSDAY, 14 JUNE 2018}

\section{What is lupus - syndrome or different entities?}

\section{SP0075 AUTOANTIBODIES - NOT ALWAYS WHAT THEY SEEM}

P.L. Meroni. Immunorheumatology Research Laboratory, IRCCS Istituto

Auxologico Italiano, 20095 Cusano Milanino, Milan, Italy

Autoantibodies (autoAbs) are the most popular and used biomarkers for systemic rheumatic diseases (SRD). AutoAbs are becoming more and more important for: i) classification (including identification of disease subtypes), ii) diagnosis, iii) prognosis, iv) monitoring activity/organ-tissue damages as well as v) response to therapy. Moreover, autoAbs have been recently used as inclusion criteria in clinical trials and their presence is a formal requisite for prescribing some new drugs. The advent of new techniques, as well as the increasing number of autoimmune diagnostic laboratories, raise the issues of assay variability and reproducibility. In addition, the new techniques need to be validated versus the standard ones in order to determine and possibly improve the correct interpretation of their results. Harmonisation of the assays for autoAbs is becoming increasingly urgent and the availability of suitable reference material for calibration and quality control is emerging as a valuable tool for increasing assay reliability. Several international committees are joining their efforts in order to improve harmonisation and at the same time to avoid misdiagnosis, unnecessary repetition of tests and ultimately to optimise patient care and costs. On the other hand, the correct interpretation of the results depends on the technique used for detecting a given autoantibody and on its own characteristics (Meroni et al. Nat Rev Rheumato.l 10:35-43; 2014). The best example is the screening tests for antinuclear antibodies (ANA). Indirect immunofluorescence (IF) ANA testing has been suggested to be the golden standard technique for ANA screening by the American College Rheumatology task force in 2010 (Meroni and Schur Ann Rheum Dis. 69:1420-2; 2010). While the sensitivity of IF ANA is high, its specificity is low as well as its post-test probability. So, the strength of a positive ANA should be evaluated in the clinical context to avoid mistakes (Mahler et al J Immunl Res. 2014:315179 2014). In addition, the isolated ANA positivity with specific staining pattern (i.e. dense fine speckled $79-$ DFS70) does not support the diagnosis of a SARD and should not be taken into consideration for both classification and inclusion criteria (Mahler et al. Autoim mun Rev. 15:198-01; 2016). IF offered advantages in comparison with the screening solid phase assays (SPA) available at the moment of the ACR position paper; however the improvement of the solid phase assays in the last years changed the situation in a substantial way. Advantages and disadvantages of the two techniques have been reviewed and discussed by several groups and none of the two tests appears to satisfy completely our needs. However, the combination of IF and SPA that include the main nuclear (cytoplasmic) antigens diagnostic for SARD, has been reported to display higher specificity and post-test probability than the use of the single tests (Bossuyt et la Ann Rheum Dis. 73:e10; 2014). New tests that employ a panel of autoantigens specific for a given subset of SARD (e.g. lupus-like, scleroderma, myositis, anti-phospholipid syndrome) are now available or are going to be launched soon increasing their specificity/post-test probability in a significant manner. For example, the combination of IF and SPA for ANA screening could decrease the risk of "false positive or negative" results, while the use of one screening assay and the new ANA profiles in the context of a specific clinical setting might increase the diagnostic power. In conclusion, our strategy to autoAb testing is changing and we should take advantage of the combination of the new serological tools for better understanding the meaning of a given positive result. The combination of the new tests will be pivotal for addressing the need for a Personalised Medicine in rheumatology.

Disclosure of Interest: P. L. Meroni Speakers bureau: Pfizer, Abbvie, INOVA Diagnostics

DOI: 10.1136/annrheumdis-2018-eular.7789

\section{THURSDAY, 14 JUNE 2018}

\section{Genetics, epigenetics and disease: is it all in the genes?}

\section{SP0076 \\ GENOMIC DISSECTION OF IMMUNE-MEDIATED DISEASE: PROGNOSIS, VASCULITIS AND EOSINOPHILS}

\section{K.G. Smith. Department of Medicine, University of Cambridge, Cambridge, UK}

Modern medicine has developed by classifying disease into defined diagnostic categories on clinical grounds. Even within a specific clinical diagnostic category however, the features of a disease and the clinical course it takes can vary greatly between individuals. I will examine two examples of how application of genomic technologies to patient cohorts has provided insight into disease behaviour.

1. A distinct biology underlying immune-mediated disease prognosis?

This presentation will explore transcriptome data that defines a clinically useful prognostic biomarker in IBD, but also addresses the specific immunological mechanisms driving long-term outcome many immune-mediated diseases (including SLE and ANCA-associated vasculitis (AAV)), and the genetics that underpins this. Evidence will be presented suggesting that the biology underlying long-term disease outcome, or prognosis, is distinct from that driving specific diagnosis, and represent an under-investigated but clinically relevant aspect of disease pathogenesis.

2. Patient stratification in ANCA-associated vasculitis: GWAS highlights new dis ease entities.

AAV has been defined using pathological criteria into granulomatosis with polyan giitis (GPA, formerly Wegener's), microscopic polyangiitis (MPA) and eosinophilic granulomatosis with polyangiitis (EGPA, or Churg-Strauss syndrome). GWAS studies performed by the European Vasculitis Genetics Consortium have revealed 4 genetically distinct syndromes within AAV - which are aligned with autoantibody specificity, and better align with clinical phenotype, patient outcome and treatment response than the traditional clinically-defined syndromes. GWAS can therefore be a tool for enhancing patient stratification, and revealing new insights into disease susceptibility and perhaps therapy.

Disclosure of Interest: None declared

DOI: 10.1136/annrheumdis-2018-eular.7822 